\title{
Health as a Human Right: A Fake News in a Post-human World?
}

\author{
Gianni Tognoni ${ }^{1} \cdot$ Alejandro Macchia $^{2}$
}

Published online: 10 November 2020

(c) Society for International Development 2020

\begin{abstract}
Based on a synthetic overview that embraces the evolution of the 'health' concept, and its related institutions, from the role of health as the main indicator of fundamental human rights - as envisaged in the Universal Declaration of Human Rights-to its qualification as the systems of disease control dependent on criteria of economic sustainability, the paper focuses on the implications and the impact of such evolution in two model scenarios which are centred on the COVID-19 pandemia. The article analyses COVID-19 both in the characteristics of its global dynamics and in its concrete management, as performed in a model medium income country, Argentina. In a world which has progressively assigned market values and goods an absolute strategic and political priority over the health needs and the rights to health of individual and peoples, the recognition of health as human right is confined to aspirational recommendations and rather hollowed out declarations of good will.
\end{abstract}

Keywords Right to health · Inequities · Post-human era · Universal Declaration of Human Rights (UDHR) · Global Burden of Diseases $\cdot$ Argentina $\cdot$ Health metrics $\cdot$ Economy $\cdot$ Universality paradigms

\section{The Need for a Conceptual Framework}

The title of an article where a question mark follows a provocative statement could easily sound as a rhetoric artefact rather than the formulation of a real research hypothesis. To provide a clearer answer to this doubt, and a full justification of the legitimacy and relevance of the question mark, let's start with a few preliminary considerations and explanatory notes, which we deem necessary as a general conceptual and methodological framework. Here they come, in this logical sequence:

- The two domains associated and confronted in the question posed in the title-public health and human rights-have been the long-term professional field of the authors. Both of them have been engaged mainly in intense research activity related to field projects and thematic areas where they have directly crossed, and experienced, the often conflicting tension and interaction between the point of view of 'health' as a fundamen-

Alejandro Macchia

alejandromacchia100@gmail.com

1 IRCCS, Ospedale Maggiore Policlinico, Milan, Italy

2 Fundación GESICA (Grupo de Estudio en Investigación Clínica en Argentina), Buenos Aires, Argentina tal human and peoples right, and that of 'health' as an increasingly dominant component of market rules. Since the Seventies, model health areas have included drug policies such as the WHO essential drugs, innovative population clinical trials, critical epidemiological use of large databases, normative regulations of accessibility to health technologies (Tognoni et al. 2019). A parallel line and a different type of research that both authors have conducted has focussed on the causes and consequences of massive violations of peoples' rights. ${ }^{1}$ Their cumulative experience, derived from the insides of these most diverse scenarios, has provided them with a solid confirmation of what has emerged with a growing consensus also in the most prestigious 'scientific' literature, in the last 10 years: structural inequality is the direct product and the expected outcome of the mainstream models of development, which trigger a highly visible impact on the rights to health and life, and prove to be a systemic source of in-human levels of inequity (Evans 2020).

- The title of the important book from Indian academic Baxi (2007) which has inspired the question mark of our contribution has proved to be neither a pessimistic forecast nor a philosophical theoretical generalization of trends on the eve of this century's first financial crisis. Health, the most sensitive indicator of the right to human

\footnotetext{
$\overline{1}$ http://permanentpeoplestribunal.org/?lang=es.
} 
dignity, has become the arena of a most impressive cultural transformation. It is the terrain that best documents the level of inequalities-iniquities generated by assuring the privilege of priority attributed to goods over humans in economic profit oriented legislations. This terrain is also an inevitable object of observation and analyses, where humans and their lives are normal, expected, quantifiable 'victims', and not inviolable subjects (for a very careful documentation of the broader evolution in this direction of juridical and institutional international scenarios (Dentico 2014).

- An independent and enriching integration of Baxi's view (with an impressive documentation from real life sociological contexts, populations, outcomes) is provided in Saskia Sassen's book which gives a most effective definition of the whys and hows of the post-human qualification. The globalization project hinges on rigid logistic and economic supply chains for whatever may be considered a market good-anything that generates profits in the selling and buying universes, including humans. The expulsion of whatever claims to be, or may be assumed to be, a 'subject' endowed with inviolable life and dignity rights (as opposed to a marketable object), is a mandatory and automatic reaction of the algorithms guiding the decisions (Sassen 2014).

- The last viewpoint that concludes this introductory framework is the most unexpected, hence the most significant one: for its peculiar origin, its world vision, and indeed its language. We refer to Pope Francis's two last encyclical documents, ${ }^{2}$ widely recognized as the expression of a truly 'universal' conversion, not confined by religious walls or political frontiers. The Pope's letters envisage humankind's re-positioning in its one and common home, that being creational nature. His approach marks an innovative recuperation of the principles of fundamental rights, which cannot be but oriented to the full inclusion of all humans (migrants, marginalized, etc.) as subjects entitled to the same dignity of life and wellbeing-which accessible health is a concrete indicator of.

\footnotetext{
${ }^{2}$ Encyclical letter Laudato Si of the Holy Father Francis. https:// www.oas.org/es/sg/casacomun/docs/papa-francesco-enciclica-lauda to-si-sp.pdf and Encyclical Letter Fratelli Tutti of the Holy Father Francis, 3rd October 2020, http://www.vatican.va/content/francesco/ en/encyclicals/documents/papa-francesco_20201003_enciclica-frate lli-tutti.html.
}

Table 1 Chronology and acronyms of fundamental international documents and events used

\begin{tabular}{lll}
\hline Acronym & Year & Meaning \\
\hline UDHR & 1948 & Universal Declaration of Human Rights \\
WHO & 1948 & World Health Organization \\
NHS-UK & 1948 & National health Service \\
UDRP & 1976 & Universal Declaration of Peoples Rights \\
TRS 615 & 1977 & WHO Technical Report Series 615, Essential \\
& & Drugs \\
NHS Italy & 1978 & National Health Service \\
PPT & 1979 & Permanent Peoples Tribunal \\
WTO & 1994 & World Trade Organization \\
GBD & 1996 & Global Burden of Diseases \\
ICC & 1998 & International Criminal Court \\
MDG & 2000 & Millennium Development Goals \\
SDH & 2008 & WHO report on Social Determinants of Health \\
UHC & 2008 & Universal Health Coverage \\
SDG & 2015 & Sustainable Development Goals \\
\hline
\end{tabular}

\section{Summarizing and Explaining A Long History Through Its 'Significant' Acronyms}

Our attempt here is to try and provide a synoptic view with some interpretative lights on the events and/or documents that have given substance to a long history. In Table 1 we intend to portray the chronology and the core themes that allow a comprehensive understanding of the challenges that we need to face in today's political, economic, conceptual scenarios while looking for an answer to the questions about the state of the right to health raised in the title of our article. The narrative of the long list's contents aims to underline the continuity and the articulation of a process where the role of the same actors (international agencies, national Governments, representatives of civil society and people's movements), and their reciprocal hierarchy of power, have mutually evolved to shape the time we are living.

As the first UN operational agency created even before the proclamation of the Universal Declaration of Human Rights (UDHR), the WHO was regarded as the immediately available, concrete and easy-to-grasp instrument to advance the political agenda of the new era, after the plight of two world wars: the human right to life and dignity could be seen and promoted as truly universal, if accessibility to the right to health could be secured. The vision then was pinning down the world to translating a declared universal principle-the right to health-into effective policies and visible practices in the context of the different countries, whose national Constitutions were meant to convert the UDHR principles into a daily experience. This horizon did not last long. Less than 30 years after the UDHR, the perspective of 
the linear development of a democratically organized society had substantially changed already. The UDPR turned out to be promoted as an independent charter to reflect and clearly denounce the dramatic limitations of the existing international law. It did not take long for the international community of States to progressively become the expression and the vehicle of 'strategic'-economic, military, political-interests, a scenario in which the multinational corporations had been playing an increasing role while denying any loyalty or accountability to the UDHR principles, and their implications.

The World Health Organization (WHO) soon became well aware of the mounting threats to its authority and field of competences, under the guise of a rapidly growing health market deprived of any control. The agency did not stay idle, in the face of what was coming. A very restricted list of 'essential drugs', based on the consensus of the scientific community, was formulated and proposed for approval to the WHO Member States. The idea was to equip governments with a tangible as well as symbolic tool to resist the pressure stemming from the neoliberal development models that mainly the International Monetary Fund (IMF) and the World Bank (WB) had started to impose.

The first half of the 1990s coincides with a major cultural and institutional translation, in the arena of health, of the shifts that had occurred to the original UDHR paradigm. The newly born World Trade Organization (WTO) declares its exclusive competence on health goods, technologies and structures. In a nutshell, the trade agenda asserts itself as the new overarching normative regime. In alliance with the WHO, the WB draws a new map of health priorities where investments should concentrate. The map does not set its priorities on the assessment of real unmet needs. It is designed on the tricky notion of the global 'economic burden' of the individual diseases (GBD), now the main-if not exclusive-term of reference of health market costs and provisions, accessibility trends, international procedures. Contrary to many expectations, the newly established International Criminal Court (ICC) declares its juridical incompetence and lack of mandate on the 'new' crimes against individual and peoples' rights committed in compliance with the new economic and trade laws.

In the narrative of the acronyms for the new millennium, health appears as one of the main goals to be pursued. Yet, the health goal is placed in a generic framework of socioeconomic variables and featured with the profile of an insurance scheme for which somebody should pay. Isn't this what the acclaimed Universal Health Coverage (UHC) approach ultimately aims for? The ambivalence, the limits, and the remarkable failures of these top-down global goals are too well known across the research reports or the administrative reviews of concerned agencies and disciplines, to require any further comment. Conversely, the silence of the scientific and institutional literature on the WHO-sponsored report on the Social Determinants of Health (SDH), is indeed very meaningful. The SDH report was supposed to remind the world, renew the memory, relaunch the era of the 'Health For All' (HFA) culture and its mobilizing manifesto, the Alma Ata Declaration. This reticence is a confirmation of how deeply the normative and epistemic changes have been interiorized. No space is left for a terminology reminiscent of the 'human' in the global reporting or goal setting, anymore. The semantic oblivion had been knowingly experimented through the GBD methodology, whose main focus is the geo-mapping of health economic burdens. The iteration of strictly descriptive and repetitive data on the growing notavoidable levels of inequalities-inequities has paved the way to the planned disappearance even from the dictionaries of health rights disciplines (Abassi 2020).

\section{From the Structural Inequalities to the COVID-19 Scenarios}

The often announced but substantially unexpected COVID19 pandemia has produced a more than foreseeable confirmation: the statistics of mortality made available for all the affected countries reproduce similar gradients of excesses of contagion and deaths which overlap and add to the underlying conditions of inequalities. A structural and pandemic characteristic featuring high income countries (HICs) just as well (Swenor 2019).

It is not the purpose of this article to either summarize or comment the rapidly changing data related to COVID-19. The main issue is rather the degree of credibility the multiple actors in the pandemic landscape - the UN agencies, the European Commission, religious leaders, academia, NGOs representing civil society, the concerned corporate actors, the science community-have vehicle through their institutional positions. The uniquely symbolic power of what has happened, its extension and consequences, cannot leave reality as it is. Radical changes in development modelling must be prepared, different hierarchies of values need to be adopted. Unfortunately, as often if the case, power balance evolution does not seem oriented to seriously engage with the challenge of radical modifications. The field of health appears to be a case in point, for its specific perfect reproduction of 'wishful thinking' strategies. A tentative check list of challenges and priorities made visible by COVID-19 would be useful.

The first and most impressive 'discovery' triggered by COVID-19 is the structural fragility of a system of marketdriven supply chains-of goods, knowledge, information, data. In the first two decades of the twenty-first century these supply-chains appeared to be the solid and untouchable protagonists of the global development models. Until the arrival 
of a biological agent, doomed to be under global knowledge's tight control, has successfully revealed the inherent ignorance and deep fragmentation defining the various branches of the most advanced disciplines of basic sciences: public health, epidemiology, health technologies.

Possibly, the unpreparedness to ignorance, in a global culture increasingly confident on the power of technology and the linearly conceived algorithmic decision-making, has produced a decisive worsening of the pandemic impact. A world proud of its digital capacity of 'linking' and 'sharing' has discovered its communication failure when it comes to real risks and potential solutions, as well as its cultural carelessness, its political and scientific lack of foresight when it comes to sharing data on unmet needs and possible solutions. Globalization's top-down approach has proved highly ineffective, 'lost in translation', when devising the needed strategies for collaborative involvement of human beings. Classical health security measures (certainly needed) have been the only remedy put in place with great variability, and unmeasurable/incomparable results in different countries and health systems. Which has entailed a recognized negative impact on the democratic and health rights of ordinary citizens, not to mention the enhanced precariousness of migrants, refugees, uninsured, homeless, simply 'poor' or marginalized people, across the world. The acclaimed capacity of handling and relying on big data to monitor, anticipate, measure the yield of the (often more than controversial) interventions has produced an international situation of confusion and uncertainty in the epidemiological understanding, and evaluation, of the pandemic evolution. Scientists and health authorities have simply failed to agree on reliable and applicable criteria for comparing their data. The retraction of scientific reports from major specialized journals, the breach of fundamental rules and practices of independent results' evaluation, even in regulatory agencies, the virtual lack of any formal coordination of public health efforts months after the inception of the 'emergency' are known elements of the daily chronicle we have familiarized with, and abundantly so.

In this scenario, the only recognized exception concerns health professionals. Confronted with the human face of the pandemic, close to the solitude of human bodies unable to breathe, medical doctors, nurses, health personnel have assured an impressive quality of presence and intensity and care across the spectrum of the different given settings. They have done so mooring their unexpected and extraordinary experience to the safe bay of the old robust-albeit tragically neglected-value of medicine as a science and practice that is grounded on a rigorous combination of professionalism, solidarity, ethics. It is reasonable to say that this "care for humans' - often institutionally unplanned, hardly politically supported, at risk now of being forgotten in terms of participation to the overall planning for the future-has been the only positive, authentic, understandable, credible form of communication to the public opinion. The other significant consensus is that health systems had suffered greatly during the pandemic crisis due to the sharp reductions of investments in human resources and community strategies, imposed by 'economic sustainability' criteria.

The scenario we have tried to summarize, therefore, bear witness to the fact that the global challenge introduced by the COVID-19 is only very partially a health problem. The ongoing economic, political, juridical 'wars' currently surrounding the narratives about the development, distribution and accessibility of the COVID-19 vaccines are, yet again, a most worrying confirmation of the real question for our societies today: namely, what model of civilization will be chosen after COVID-19 (Bloom et al. 2020; Nowak et al. 2020). Humans - their lives and their values of democracy, solidarity, and hope for a better future-have played their role as victims, rather than as subjects. The viral pandemic has disclosed the structural pandemic impact of the social and economic viruses that have infected the global chains of knowledge production, denied the possibility of delivering the common goods, seconded the vested priorities of private powerful minorities over the vast majorities of peoples.

The most recent reports from the GBD groups have 'discovered' that the global data which consistently document the extent and the severity of the impact of the inequalities worldwide should evolve from a strategy of neutral description into one approach of looking into the avoidability of such inequalities-iniquities. It seems an obvious cultural and methodological change, but it could represent quite a revolutionary step forward from the tragically impressive analytical model illustrated by Burstein et al. in a top scientific journal (2019). A substantial proportion of the 123 million neonatal, infant, paediatric deaths occurred in the twenty-first century could be considered avoidable with simple, mainly non-sanitary measures, the study says. Nothing else. Ultimately, there in an urgent need for re-establishing human lives and deaths as the measure of needs and outcomes, against the persistently dominant focus on disease burdens.

\section{A Southern Perspective from Argentina}

Repeating the usual soliloquy of 'international' literature, an abundant production of medical articles has flooded physicians and public health professionals working in the Global South with the intent of explaining them what is happening in their countries, what their needs are, what lessons they should learn from the COVID-19 pandemic. While the flow of information is meant to be educational, the appropriate term we can use is, possibly, indoctrination (Daniels 2020; Hogan et al. 2020; Walker et al. 2020). With COVID-19, 
a new wave of top-down, complex and unverifiable mathematical models keep coming from the center of the world foreseeing when the peripheries will run out of respirators, how many people will die in the next semester, how the pandemic will impact tuberculosis and HIV infected people (Walker et al. 2020), and how all this knowledge should inform a model of healthcare organization (Walker et al. 2020). All data must be noted and lessons learnt.

This dynamic was not brought in by COVID. For decades, centrally produced guidelines have indicated how to invest mostly borrowed money for the 'proper' administration of health resources. To verify the sparkle of the truth hidden in the lies, a small, basic technical frame of reference could be useful to discuss concepts related to solidarity, rights and autonomies of real populations. For decades now (World Bank 1993; Murray and Lopez 1996; Cooper et al. 1998; Jamison et al. 2006; Mathers and Loncar 2006), GBD reports have informed us that in Argentina things are going reasonably well. The increase in life expectancy, the decrease in infant mortality and the overall improvement in access to healthcare (HAQ Index) indicate that things have improved significantly. The Institute of Health Metrics (its logo explicitly states: 'measuring what matters') incorporates comparisons with other countries to provide a reference aimed at defining the magnitude of trends and problems. This approach makes Argentina indistinguishable from Bosnia and Herzegovina when it comes to deaths from cirrhosis, although it would profile it better than China in terms of self-inflicted injuries and significantly worse than the Mauritius Islands of East Africa in neonatal conditions. This is a description of what is published.

Beyond the international metrics and their grotesque comparisons, these estimates lead to an important conceptual distortion. When analyzing Argentina's factual data, we see that the average improvement occurs at the expenses of a fraction of the population. Inequity increases and access to services worsens among those who need it most (Macchia et al. forthcoming). Women, for example. Their health situation is dramatic. Available data from the last 2 years show that in 2016-2017 Argentinian women have the same standardized death rate as in 1990-1991. Premature mortality increases significantly among the poorest segments of society, and does so with particularly harsh trends among women over 60 (Macchia et al. forthcoming). Yet, these populations disappear completely from the health metrics analysis. Their eclipse is not accidental. The categorization of a wide, polychromatic and heterogeneous universe into smaller, measurable, homogeneous fractions recording an average improvement, manages to reduce the world to estimates showing some improvement but leaving out immense population groups (Sassen 2014). The resulting disappearance of humans portions shrinks the world to a selected group of inhabitants, those who coincide with as many given categories or clusters.

The COVID is a chapter written with the same letters. The grotesque comparisons and 'rankings' among countries circulated by the media on a daily basis, arithmetically counting the number of sick and dead, only represent the further trivialization of a culture that does not pretend to learn or listen but to talk. In a world where many could know so much, the number of people who know something seriously is alarmingly low. Again, a few technical data to reflect on this.

In Buenos Aires, a city of 3.1 million people, the cumulative incidence rate (IC95\%) of COVID-19 was 1890 (18321948) per 100,000 people, as of August 2020. In the slums it was 5916 (5542-6289) corresponding to 13,829 people, while in the city areas not inhabited by proximity to the slums it was 1559 (1505-1612) corresponding to 44,314 people. Despite their younger age, slum dwellers have a significantly higher mortality rate. Even disaggregating by age, sex and risk factors, the mortality rate of slum population doubles that of people living outside the slums (Macchia et al. submitted). Ultimately, COVID-19, like coronary disease, tuberculosis, malnutrition, obesity and cancer (Marmot $2005)$ is socially conditioned.

Additionally, the possibility of confining cases and close contacts in overcrowded housing is close to zero. In Buenos Aires more than $60 \%$ of COVID-related cases among slums dwellers had to be confined to alternative care sites (hotels). ${ }^{3}$ What's the point of describing COVID in Argentina merely counting the number of cases, without telling people's stories, without understanding the different human groups, their identities and challenges? It seems that global world standards do not need this information. After all, slum dwellers are not part of the global picture, just like the over one billion world inhabitants who live undocumented (Byass et al. 2013), and who spend their lives in daily statistical and political anonymity.

\section{The Right to Health and his Opponents}

Health has ceased to be a public matter since years. It was regarded as such for a brief period of political history, a time that cannot return it seems, at least not in the short term. But it is not only the administration of health care that has been transferred into private hands. Public health research and its priorities setting is in private hands, too.

\footnotetext{
$330 \%$ of COVID cases in Buenos Aires (60\% of cases located in slums) were housed in Alternative care Sites (ACS) which are hotels converted into assistance centres for low-risk people. This was done because the infection rate is so high in the slums that if only $50 \%$ of the patients were admitted, the health system would collapse.
} 
Philanthrocapitalism, particularly through the Bill \& Melinda Gates Foundation, finances the research agenda and modulates collective health's decisions at the level of the multilateral community. Members of 'philanthropic' foundations have close ties to pharmaceutical companies, so they sail from private interest to public policymaking with no restraints. Philanthropic foundations' staff sits on corporate entities' boards and public health organizations alike, as if this were a normal circumstance (Birn 2014). In COVID's case, the relationship between the director of the 'Warp Speed' programme and his relationship with one of the candidates to produce the vaccine is one point in case. example. $^{4}$

The relationship between health, collective rights and market interests has become increasingly blurred. Some Latin American countries hailed the vaccine production in their own territories, announcing it as the exercise of state responsibility for the benefit of collective health. But this narrative is nowhere close to reality, at least in Argentina. The production of the AstraZeneca and the Oxford University vaccine was entrusted by the consortium to an Argentine businessman who owns a group of transnational companies. The business group quickly clarified that this is an 'agreement between private parties' and that the Argentinian state has nothing to do with it. In fact, the Argentinian State does have a role. It has secured the advanced purchase of tens of millions of doses without even negotiating the price of the product. It takes the risk of vaccinating early, in the absence of long-term safety monitoring results. But there's more. In the past, the government has financed the vaccine production plants. The state is indeed playing a role with a variety of interventions, including that of a consumer. ${ }^{5}$ The race to win the market competition encouraged by ignorant politicians with no scruples is doomed to produce even greater damage (Krause et al. 2020). Obtaining a poorly effective vaccine (as it will most likely be the case for the first generation of products) will generate a gold standard that other follow-on trials will adopt as their benchmark.

\footnotetext{
${ }^{4}$ Documents Reveal Potential Unresolved Conflicts Of Interest Among Top Operation Warp Speed Advisors. https://coronaviru s.house.gov/news/press-releases/documents-reveal-potential-unres olved-conflicts-interest-among-top-operation; Elizabeth Warren, others seek details of Warp Speed co-chief Moncef Slaoui's contract, https://www.washingtonpost.com/business/2020/08/25/operationwarp-speed-moncef-slaoui-contract-ethics/; On the road with Operation Warp Speed, the U.S. COVID-19 vaccine effort, https://www. sciencemag.org/news/2020/09/road-operation-warp-speed-us-covid19-vaccine-effort.

5 On 18 October 2020, the minister of Health has assured that in March 2021 a massive vaccination campaign will be carried out in Argentina with the Astra Zeneca vaccine, the production of which is planned in Argentina available at https://www.lanacion.com.ar/polit ica/coronavirus-argentina-gines-gonzalez-garcia-en-marzo-nid24 83167.
}

In 1935, Martin Heidegger envisaged what did not exist then, but we must consider today: 'When the farthest corner of the globe has been conquered technologically and can be exploited economically; when any incident you like, in any place you like, at any time you like, becomes accessible as fast as you like; when you can simultaneously 'experience' an assassination attempt against a king in France and a symphony concert in Tokyo; when time is nothing but speed, instantaneity, and simultaneity, and time as history have vanished from all Being of all peoples; when a boxer counts as the great man of a people; when the tallies of millions at mass meetings are a triumph; then, yes then, there still looms like a specter over all this uproar the question: what for?-where to?-and what then?'

\section{Conclusions}

The substantial convergence between the initial question of our title and the questions that Heidegger formulates in the quote we have chosen could be seen as the most coherent and certainly worrying conclusion.

The historical parenthesis when the universality of human rights was politically adopted not as an utopia, but as an innovative (first in history!), concrete, long-term programme of a post-war (never again!) world order is radically threatened, if nor formally declared obsolete. The ongoing COVID-19 event is the symbolic-even more importantly than the real-expression of a time when unmet needs and questions come forcefully to the fore as protagonists, higher and above the accepted challenge. Health was the first ideal and realistic proposition of that historical parenthesis (Table 1): the life with dignity of human beings (no one left behind!) was assumed to be the mandatory, reasonable, long term outcome. But the amazing successes of life sciences in the last decades have moved in parallel with the progressive transformation of the economy from a valuebased, competent science that guided the use of resources as a key component to support human development, into an autonomous uncontrolled power. The legitimate and binding indicators of human and peoples' rights were marginalized, to the advantage of development models and economic strategies, whereby market goods acquired the status of free and untouchable subjects of legal contractual laws.

On the other hand, most juridical sciences and institutions, satisfied with the formal solidity of their principles, conventions and constitutions based on universality paradigms, became confined to a role of arbiters and controllers of the new global order 's compliance. In this scenario, the universality of human and peoples' rights was progressively transformed into a dependent variable of economic sustainability and of the impunity needed by the new political and 
economic powers, active at the national and international level.

The 2020 pandemic, triggered by one of the oldest companions-enemies of human life and history, could become a determinant of a cultural, social, mental lockdown; a test of the limits of resistance of societies, more than a powerful stimulus of resilience. As representatives and disenchanted actors of disciplines where uncertainties and failures compel to imagine, promote, experiment answers 'to care for the right of life' - this indeed has been the only recognized 'light' of engagement in the dark tunnel of the pandemicwe have the duty to give our last question an horizon. Something that does not exist but has a very important role, as Eduardo Galeano showed in his imagined upside-down world. The role of the horizon is simply to oblige us to move on and go ahead. Let's at least be the custodians, let's at least preserve the memory of the utopia of the first acronyms in Table 1. The universal right to life in dignity is assumed in a bottom-up mobilization by the subjects of history, the 'discarded', the expelled majorities, as a priority area of high cultural and social conflicts, to become the political terrain of struggle and confrontation with the economic and institutional powerful minorities, in the quest for a new, human, parenthesis. It is our horizon. Not an answer.

\section{References}

Abassi, Kamran. 2020. Health inequalities: Death by political means. British Medical Journal 368: m755.

Baxi, Upendra. 2007. Human Rights in a Post Human World: Critical Essays. Oxford: Oxford University Press.

Birn, Anne-Emanuelle. 2014. Philanthrocapitalism, past and present: The Rockefeller Foundation, the Gates Foundation, and the setting(s) of the international/global health agenda. Hypothesis 12 (1): e8. https://doi.org/10.5779/hypothesis.v12i1.229.

Bloom, Barry R., Geln J. Nowak, and Walter Orenstein. 2020. When Will We Have a Vaccine? Understanding Questions and Answers about Covid-19 Vaccination. The New England Journal of Medicine. https://doi.org/10.1056/nejmp2025331.

Burstein, Roy, Nathaniel J. Henry, Michael L. Collison, et al. 2019. Mapping 123 million neonatal, infant and child deaths between 2000 and 2017. Nature 574: 353-358.

Byass, Peter, Maximilian de Courten, Wendy J. Graham, Lucie Laflamme, Affette McCaw-Binns, Osman A. Sankoh, Stephen M. Tollman, and Basia Zaba. 2013. Reflections on the Global Burden of Disease 2010 Estimates. PLoS Medicine 10 (7): e1001477. https://doi.org/10.1371/journal.pmed.1001477.

Cooper, Richard S., Babatunde Osotimehin, Jay S. Kaufman, and Terrence Forrester. 1998. Disease burden in sub-Saharan Africa: What should we conclude in the absence of data? The Lancet 351: 208-210

Daniels, Joe Parkin. 2020. COVID-19 cases surge in Colombia. The Lancet 396: 227.

Dentico, Nicoletta. 2014. Nutrition, Pathologies of Power and the Need for Health Democracy. Development 57 (2): 184-191.
Evans, Michele K. 2020. Health Equity: Are We Finally on the Edge of a New Frontier? The New England Journal of Medicine 383: 997-999.

Hogan, Alexandra B., Britta L. Jewell, Ellie Sherrard-Smith, et al. 2020. Potential impact of the COVID-19 pandemic on HIV, tuberculosis, and malaria in low-income and middle-income countries: a modelling study. The Lancet Global Health 8: e1132-e1141.

Jamison, Dean T., Joel G. Breman, Anthony R. Measham, George Alleyne, Mariam Claeson, David B. Evans, Prabhat Jha, Anne Mills, and Philip Musgrove (eds.). 2006. Disease control priorities in developing countries. 2nd ed. New York: Oxford University Press. http://dcp-3.org/dcp2. Accessed 25 September 2020.

Krause, Philip, Thomas R. Fleming, Ira Longini, Ana Maria Henaorestrepo, and Richard Peto. 2020. COVID-19 vaccine trials should seek worthwhile efficacy. The Lancet 396 (10253): 741-743.

Macchia, Alejandro, J. Mariani, D. Nul, H. Grancelli, Gianni Tognoni, and H.C. Doval. forthcoming press. An analysis of death trends in Argentina, 1990-2017, with emphasis on the effects of economic crises. JOGH.

Macchia, Alejandro, D. Ferrante, G. Battistella, Mariani, and F. González Bernaldo de Quirós. submitted. COVID-19 among the inhabitants of the slums in the city of Buenos Aires: A population based study. BMJ Open.

Marmot, Michael. 2005. Social determinants of health inequalities. The Lancet 365: 1099-1104.

Mathers, Colin D., and Dejan Loncar. 2006. Projections of global mortality and burden of disease from 2002 to 2030. PLoS Medicine. 3(11): e442 https://journals.plos.org/plosmedicine/artic le? $\mathrm{id}=10.1371$ /journal.pmed.0030442. Accessed 25 September 2020.

Murray, Christopher J.L., and Alan D. Lopez (eds.). 1996. The global burden of disease: a comprehensive assessment of mortality and disability from diseases, injuries and risk factors in 1990 and projected to 2020. Cambridge: Harvard University Press on behalf of the World Health Organization and the World Bank.

Nowak, Glen J., Emilie Karafillakis, and Heidi Larson. 2020. Pandemic influenza vaccines: Communication of benefits, risks, and uncertainties. In Communicating about risks and safe use of medicines: Real life and applied research, ed. P. Bahri, 163-178. Singapore: Springer Nature.

Sassen, Saskia. 2014. Expulsions: Brutality and Complexity in the Global Economy. Cambridge: Harvard University Press.

Swenor, Bonnielin. 2019. Meeks LM, Disability Inclusion. Moving beyond mission Statements. The New England Journal of Medicine 380: 2089-2091.

Tognoni, Gianni, Maria Grazia Franzosi, and Silvio Garattini. 2019. Embedding patient- and public health-oriented research in a national health service: The GISSI experience. Journal of the Royal Society of Medicine 112 (5): 200-204.

Walker, Patrick G.T., Charles Whittaker, Oliver J. Watson et al. 2020. The impact of COVID-19 and strategies for Mitigation and suppression in low- and middle-income countries. Science, 369(6502): 413-422. https://doi.org/10.1126/science.abc0035.

World Bank. 1993. World Development Report 1993. Investing in health. New York: Oxford University Press for the World Bank.

Publisher's Note Springer Nature remains neutral with regard to jurisdictional claims in published maps and institutional affiliations. 